\title{
Chondrosarcoma of the Nasal Septum-A Rare Subsite: Case Report with Review of Literature
}

\author{
Nidhi Gupta10 ${ }^{10}$ Awadhesh Kumar Pandey ${ }^{1}$ Kislay Dimri ${ }^{1} \quad$ Surinder K Singhal ${ }^{2}$ Neeraj Rathee ${ }^{1}$ \\ Shalu Naryal ${ }^{1}$ Pooja Sharma ${ }^{3}$ Amitabh Kumar Upadhyay ${ }^{4}$ \\ ${ }^{1}$ Department of Radiation Oncology, Government Medical College \\ and Hospital, Chandigarh, India \\ 2 Department of ENT, Government Medical College and Hospital, \\ Chandigarh, India \\ ${ }^{3}$ Department of Pathology, Government Medical College and \\ Hospital, Chandigarh, India \\ ${ }^{4}$ Department of Medical Oncology, Tata Main Hospital, Jamshedpur, \\ Jharkhand, India \\ Ind J Med Paediatr Oncol 2021;42:506-509. \\ Address for correspondence Nidhi Gupta, MD, DNB, Department of \\ Radiation Oncology, Government Medical College and Hospital, \\ Sector 32, Chandigarh 160030, India \\ (e-mail: nidhiguptaonco@gmail.com).
}

\begin{abstract}
Keywords

- malignant

- cartilage

- head and neck

- grade

- treatment

Chondrosarcomas are the second most common primary malignant bone tumors. Head and neck chondrosarcomas constitute less than $10 \%$ of these tumors, rarely arising from the nasal septum. These are locally aggressive malignant tumors arising from the cartilaginous framework of the nasal septum. Rarity of the tumor coupled with nonspecific symptoms makes it a diagnostic dilemma. Diagnosis requires endoscopy, radiology, and final histopathology for confirmation. Treatment is mainly surgical, requiring complete surgical excision with clear margins. Radiation has a role in unresectable tumors or for tumors with positive margins after surgery. Survival depends on the grade of tumor that predicts the metastatic potential of the tumor. We present a rare case of chondrosarcoma arising from the nasal septum in a 29-yearold young female presenting with complaints of nasal obstruction. Computed tomography was suggestive of a calcified cartilaginous tumor arising from the nasal septum. Endoscopic excision was done and postoperative histopathology showed grade II chondrosarcoma with clear margins. No adjuvant treatment was given to our patient and 2 years post-excision patient is disease free.

To conclude, chondrosarcoma of the nasal septum is a rare tumor, with nonspecific symptoms. Surgery with clear margins remains the treatment of choice. Prognosis depends on the extent of tumor at presentation, resection margins, and grade of tumor.
\end{abstract}

DOI https://doi.org/ 10.1055/s-0041-1736210. ISSN 0971-5851.
(C) 2021. Indian Society of Medical and Paediatric Oncology. All rights reserved.

This is an open access article published by Thieme under the terms of the Creative Commons Attribution-NonDerivative-NonCommercial-License, permitting copying and reproduction so long as the original work is given appropriate credit. Contents may not be used for commercial purposes, or adapted, remixed, transformed or built upon. (https://creativecommons.org/ licenses/by-nc-nd/4.0/)

Thieme Medical and Scientific Publishers Pvt. Ltd., A-12, 2nd Floor, Sector 2, Noida-201301 UP, India 


\section{Introduction}

Chondrosarcomas are characterized as slow-growing malignant tumors that arise from cartilaginous structures. Very few of these $(5-10 \%)$ are found in the head and neck, commonly in the maxilla and mandible. ${ }^{1}$ Within head and neck chondrosarcomas, tumor arising from the nasal septum is rare and usually reported as extensive tumors of sinonasal structures, extending to sinuses, nasopharynx, hard palate, orbit or base of skull with difficulty in identifying the origin of tumor. Tumors with localized and isolated involvement of nasal septum are rare. ${ }^{2-4}$ These tumors most commonly present in the middle age. ${ }^{5}$ Etiopathogenesis remains unknown. Diagnosis remains difficult as patients present with nonspecific symptoms. Treatment of choice is surgical resection. Radiation treatment is limited for selected cases. ${ }^{1,3}$ We present a rare case of localized chondrosarcoma arising from nasal septum. The clinical symptoms, radiological findings, pathological findings, treatment and review of literature are also discussed.

\section{Case Report}

A 29-year-old female presented with complaints of left-side nasal obstruction for the last 3 years, not relieved with medication. It was associated with postnasal discharge and occasional pain in nasal cavity (left). It was also associated with snoring while sleeping.

On examination, external framework of the nose was normal, a single diffuse swelling was present $2 \times 2 \mathrm{~cm}$ over left side dorsum of nose over the middle one-third, and overlying skin was normal. Right nasal septum was deviated, multilobar soft tissue mass was present over the left side of nasal cavity, obscuring whole of nasal cavity, soft tissue mass was also present over the right-side floor, not completely obscuring nasal cavity.

Computed tomography scan of paranasal sinuses ( - Fig. 1) showed large expansile mass arising from the nasal septum

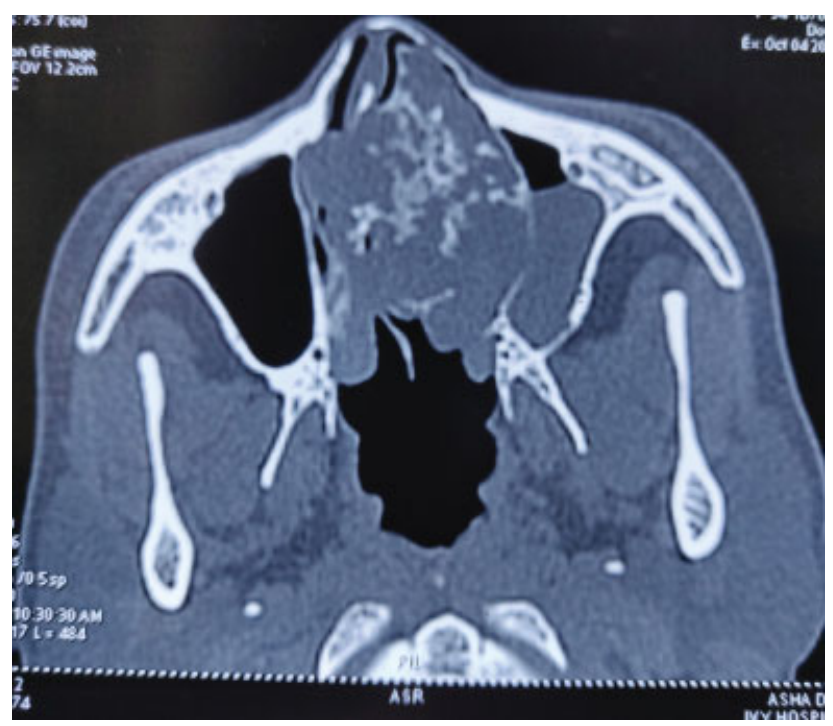

Fig. 1 Preoperative computed tomographic image.

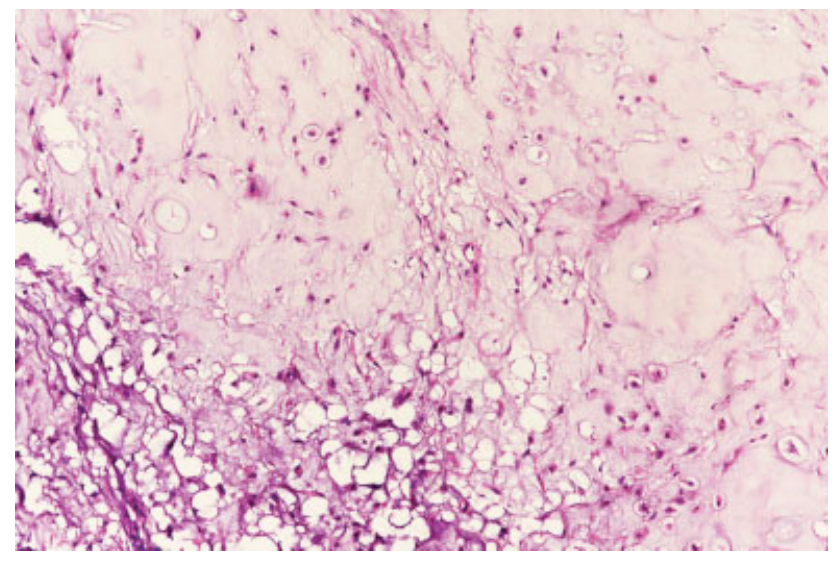

Fig. 2 Postoperative histopathology.

filling the entire left and most of the right nasal cavity, measuring $5.5 \times 4.6 \times 3.8 \mathrm{~cm}$ and showing multiple calcified areas within the mass suggestive of cartilaginous tumor.

Patient underwent nasal endoscopy that showed lobulated pinkish mass present in left nasal cavity in anterior part, firm, gritty, did not bleed on touch. Mass was also present on the right side arising from the septum. Mass was well circumscribed and pressing on the lateral wall. However, planes with the lateral wall were well defined.

In view of the above, the patient underwent endoscopic excision of the tumor mass. Taking adequate margins around the origin of tumor (septal cartilage), the whole tumor was removed in toto along with the septal cartilage.

Postop histopathology report was suggestive of grade II chondrosarcoma (-Fig. 2) with all resection margins free of tumor; the closest margin was $0.2 \mathrm{~cm}$ away. In view of clear margins, patient required no adjuvant treatment and was kept on follow-up. Two years post-treatment, the patient is disease free.

\section{Discussion}

Chondrosarcoma is a slow-growing nonepithelial malignant tumor of the bone that produces cartilaginous matrix. Most commonly, they arise from the long bones, pelvis, ribs, scapula, and sternum. Head and neck chondrosarcomas comprise hardly 5 to $10 \%$ cases. ${ }^{1,3,4}$ Sinonasal cancers comprise $3 \%$ of all head and neck cancers that usually present as advanced disease with involvement of sinuses and nasal cavity simultaneously. Isolated origin of primary chondrosarcoma from nasal septum is found to be an extremely rare condition. $^{2}$

The most common age at presentation for chondrosarcomas as reported in literature is between 40 and 60 years, ${ }^{5}$ and our patient was 29 years old, which increase the rarity of the case. Chondrosarcomas have a male preponderance and our patient was a female. ${ }^{6}$

The most common presenting symptom for sinonasal tumors is nasal obstruction and our patient also presented with a similar complaint. Other symptoms may include epistaxis, chronic nasal discharge, headache, vision deterioration, or symptomatic cranial nerve involvement in 
advanced cases. ${ }^{3,5}$ The slow-growing nature of the chondrosarcomas results in large tumors at presentation. Differential diagnosis for cartilaginous tumor from septal sites includes inverted papilloma, schwannoma, squamous cell carcinoma, adenocarcinoma, sinonasal melanoma, and minor salivary gland tumor. ${ }^{3,5}$

Chondrosarcomas are tumors of mesenchymal origin with malignant behavior. The spectrum of these tumors may vary, either low-grade tumors that are indolent to high-grade tumors that are very aggressive. Histopathologically, they are characterized by cellular, poorly differentiated, mesenchymal cells mixed with small areas of well-differentiated cartilage, which looks benign. Grading of the tumors is based on rate of mitosis, cellularity, and nuclear size. ${ }^{2}$ Based on these findings, the tumor can be graded into three grades I, II, and III ranging from well differentiated to poorly differentiated tumors. The size and grade of the tumor indicate the local aggressiveness, chances of metastases, and survival outcomes. Grade is the most common prognostic indicator. ${ }^{7}$ Our patient had an intermediate grade tumor on the postoperative histopathology. Histological differentials include chondroid differentiation in osteosarcomas, enchondromas, and chondroid chordomas. Immunohistochemical staining is useful for confirmation of chondrosarcomas as they show positive immunoreactivity to cytokeratin and S100 protein. ${ }^{7}$

Radiologically, chondrosarcomas are characterized by soft tissue multilobulated expanding mass with areas of nodular and plaque like calcification. On computed tomography, the characteristic findings include presence of chondroid matrix with peripheral and central calcifications, which are scattered. Erosion of the septum and surrounding bony structures is common. ${ }^{8}$ On magnetic resonance imaging (MRI), the periphery of the tumor shows contrast enhancement, while the central core that is chondromatous does not enhance. Soft tissue details, intracranial and intraorbital extensions are better appreciated on MRI. ${ }^{1,8}$ The typical calcifications in the cartilaginous tumor associated with chondrosarcoma were also seen in our patient as enclosed in the radiology images. 18-fluoro-2-deoxyglucose positron emission tomography (18F-FDG PET) helps in the tumor grading of the cartilaginous tumor and hence predicts the outcomes. In a study by Feldman et al, maximum standardized uptake value cutoff used to distinguish benign and malignant cartilage neoplasms in 26 operated cartilaginous tumors was 2.0. In this study, sensitivity of 18F-FDG PET was $90.9 \%$, specificity was $100 \%$, and accuracy was $96.6 \% .{ }^{9}$ Knowledge of the radiological findings can help to preoperatively confirm the diagnosis and plan a surgical resection accordingly.

Treatment of choice for chondrosarcomas remains surgical resection with wide margins. ${ }^{1,3}$ The recurrence rate associated with positive margins is as high as $65 \%{ }^{8}$ Surgical approaches that can be used for resection include WeberFerguson, lateral rhinotomy, sublabial transnasal, Le Forte I downfracture, anterior craniofacial approach, and transnasal excision. Endoscopic resection can be preferred for lesions limited to the nasal cavity, without evidence of intracranial, base of skull, or orbital extension. ${ }^{7,8}$ In view of the limited extent of the tumor in our patient, she also underwent endoscopic resection. Endoscopic surgery has several advantages, as it provides better magnification, illumination, better cosmetic outcomes, shorter operative time, and superior visualization facilitating complete endonasal resection of the tumor. Added advantages of endonasal resection include preservation of the physiological functions of the sinonasal structures and mucociliary clearance. Morbidity is less and hospital stay is minimized. ${ }^{7,8}$

Chondrosarcomas have generally been considered less radiosensitive in view of their indolent nature; however, radiotherapy is indicated for unresectable tumor involving vital structures, recurrent cases, or as adjuvant for positive surgical margins and high-grade histology. ${ }^{3}$ Radiation by proton therapy should be preferred for such cases. ${ }^{10}$ Radiation doses greater than $60 \mathrm{~Gy}$ should be delivered with conformal techniques. ${ }^{11}$ Chemotherapy has no proven role for these tumors. ${ }^{1,3}$

Prognosis depends on extent of the tumor, extent of resection, and grade of tumor. ${ }^{12}$ The 5 -year survival rate, when all grades of chondrosarcoma are included, ranges from 54 to $81 \%{ }^{13}$ Regular lifelong follow-up with nasal endoscopy is required to rule out recurrence. At 2 years of follow-up, our patient is disease free.

\section{Conclusion}

Chondrosarcoma of the nasal septum is an extremely rare presentation for head and neck tumors. The nonspecific symptoms pose a diagnostic dilemma for the treating physician. Combination of diagnostic endoscopy, radiology, and histology nails the diagnosis. Treatment is primarily surgical with minimally invasive techniques decreasing the morbidity. Adjuvant treatment like radiation is reserved for patients with incomplete resection or high-grade tumors. Prognosis and outcome depend on the grade of the tumor. The prognosis is excellent for indolent tumors.

Declaration of Patient Consent

Witten informed consent was taken from the patient.

Authors' Contribution

\begin{tabular}{|l|l|}
\hline $\begin{array}{l}\text { Name of authors } \\
\text { (corresponding } \\
\text { author first) }\end{array}$ & Author's contribution \\
\hline Nidhi Gupta & $\begin{array}{l}\text { Designed study, collected data, } \\
\text { analyzed, and wrote the first draft of } \\
\text { manuscript }\end{array}$ \\
\hline Awadhesh K Pandey & Analyzed and edited the manuscript \\
\hline Kislay Dimri & Analyzed and edited the manuscript \\
\hline Surinder K Singhal & $\begin{array}{l}\text { Designed study and edited the } \\
\text { manuscript }\end{array}$ \\
\hline Neeraj Rathee & Collected data \\
\hline Shalu Naryal & Collected data \\
\hline Pooja Sharma & Collected data \\
\hline
\end{tabular}




\section{Financial Support}

Nil.

\section{Conflicts of Interest}

Nil.

\section{References}

1 Stavrakas M, Nixon I, Andi K, et al. Head and neck sarcomas: clinical and histopathological presentation, treatment modalities, and outcomes. J Laryngol Otol 2016;130(09):850-859

2 Magnano M, Boffano P, Machetta G, Garibaldi E, Delmastro E, Gabriele P. Chondrosarcoma of the nasal septum. Eur Arch Otorhinolaryngol 2015;272(03):765-772

3 Khan MN, Husain Q, Kanumuri VV, et al. Management of sinonasal chondrosarcoma: a systematic review of 161 patients. Int Forum Allergy Rhinol 2013;3(08):670-677

4 Belgioia L, Vaccara EM, Bacigalupo A, Corvò R Management of nasal septum chondrosarcoma occurring in elderly: a case report. Cureus 2017;9(07):e1497

5 Bahgat M, Bahgat Y, Bahgat A, Elwany Y. Chondrosarcoma of the nasal septum. BMJ Case Rep 2012;2012:bcr2012006266

6 Bulut F, Kizilay A, Aydin NE. Chondrosarcoma of the nasal septum: a case report. Kulak Burun Bogaz Ihtis Derg 2004;12(1-2):39-41
7 Downey TJ, Clark SK, Moore DW. Chondrosarcoma of the nasal septum. Otolaryngol Head Neck Surg 2001;125(01):98-100

8 Rassekh CH, Nuss DW, Kapadia SB, Curtin HD, Weissman JL, Janecka IP. Chondrosarcoma of the nasal septum: skull base imaging and clinicopathologic correlation. Otolaryngol Head Neck Surg 1996;115(01):29-37

9 Feldman F, Van Heertum R, Saxena C, Parisien M. 18FDG-PET applications for cartilage neoplasms. Skeletal Radiol 2005;34(07): 367-374

10 Rieken S, Habermehl D, Nikoghosyan A, et al. Assessment of early toxicity and response in patients treated with proton and carbon ion therapy at the Heidelberg ion therapy center using the raster scanning technique. Int J Radiat Oncol Biol Phys 2011;81(05): e793-e801

11 Kamal AF, Husodo K, Prabowo Y, Hutagalung EU. Correlation between survival and tumour characteristics in patients with chondrosarcoma. J Orthop Surg (Hong Kong) 2015;23(03): 365-369

12 Coca-Pelaz A, Rodrigo JP, Triantafyllou A, et al. Chondrosarcomas of the head and neck. Eur Arch Otorhinolaryngol 2014;271(10): 2601-2609

13 Kaufman JK, Pritz MB, Righi PD, Bizal JC. Craniofacial resection of a nasoseptal chondrosarcoma: case report and review of the literature. Surg Neurol 1999;52(03):265-268, discussion 268-269 